\title{
Patient characteristics, adherence, and costs of oral anticoagulation therapy in non-valvular atrial fibrillation using the Dubai Real-World Claims Database
}

\author{
Moutaz El Kadri, Ahmed Ghorab ${ }^{1}$, Jean Joury ${ }^{1}$, Mohamed Farghaly², Nancy Awad ${ }^{3}$, \\ Badarinath Chickballapur Ramachandrachar ${ }^{3}$, Ashok Natarajan ${ }^{3}$

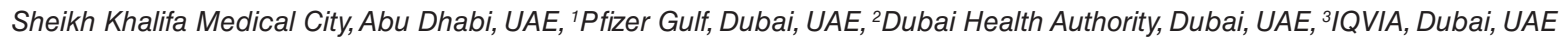

\begin{tabular}{|c|}
\hline Access this article online \\
\hline Website: www.avicennajmed.com \\
\hline DOI: 10.4103/ajm.ajm_228_20 \\
\hline Quick Response Code: \\
\hline$\square$ \\
\hline
\end{tabular}

\begin{abstract}
Background: Non-vitamin $\mathrm{K}$ antagonist oral anticoagulants (NOACs) reduce the risk of stroke in patients with non-valvular atrial fibrillation (NVAF) and have better safety profile than vitamin $\mathrm{K}$ antagonists (VKAs). However, there is a dearth of quality, real-world, patient data on the use of these drugs to guide healthcare policies in United Arab Emirates (UAE). Aims and Objectives: The aim is to address the knowledge gap in demographic and clinical profiles of NVAF patients on NOACs (apixaban, rivaroxaban, and dabigatran) and warfarin in UAE. Materials and Methods: This retrospective cohort analysis utilized the Dubai Real-World Claims Database to extract anonymized longitudinal data on NVAF patients with at least one NOAC or warfarin claim between January 2015 and March 2019. Data examined included comorbidities, healthcare resource utilization (HCRU), treatment adherence, and clinical events. Results: From 11,086 NVAF patients in the database, 940 patients on oral anticoagulant treatment were selected with mean age of $58.6 \pm 14.7$ years and $73.7 \%$ men. At baseline, the mean $\mathrm{CHA}_{2} \mathrm{DS}_{2}$-VASc risk score was 2.4 , and the mean Deyo-Charlson comorbidity index $(\mathrm{CCl})$ score was 1.6 . Most patients $(71 \%)$ started oral anticoagulation treatment on a standard index dose. High medication possession ratio (MPR) and proportion of days covered (PDC) were observed in $86.8 \%$ and $43.1 \%$ of the overall cohort. The mean number of HCRU claims and cost during the 180-day follow-up period was 18.5 and 9,747 USD, respectively. Warfarin users accounted for both the highest number of claims and cost, whereas apixaban accounted for the lowest figures. Time to first major bleeding was shorter for warfarin users compared with patients on NOACs. Longer times to first stroke/systemic embolism (SE) were observed for rivaroxaban and warfarin. Conclusion: This study provides important comparative insights about comorbidities, adherence, HCRU, and outcome events among NOAC and warfarin users from real-world clinical practice settings.
\end{abstract}

Key words: Anticoagulants, atrial fibrillation, claims, healthcare resource utilization, NOAC, real-world study

\section{INTRODUCTION}

Vitamin K antagonists (VKAs), especially warfarin, have long been used as oral anticoagulants (OACs) for prevention of stroke and systemic embolism (SE) in

Address for correspondence: Dr Moutaz El Kadri,

Sheikh Khalifa Medical City, P. O. Box 51900, Abu Dhabi, UAE.

E-mail: mkadri@seha.ae
This is an open access journal, and articles are distributed under the terms of the Creative Commons Attribution-NonCommercial-ShareAlike 4.0 License, which allows others to remix, tweak, and build upon the work non-commercially, as long as appropriate credit is given and the new creations are licensed under the identical terms.

For reprints contact: reprints@ @medknow.com

Cite this article as: El Kadri M, Ghorab A, Joury J, Farghaly M, Awad N, Ramachandrachar BC, et al. Patient characteristics, adherence and costs of oral anticoagulation therapy in non-valvular atrial fibrillation using the Dubai Real-World Claims Database. Avicenna J Med 2021;11:93-102. 
patients with non-valvular atrial fibrillation (NVAF). ${ }^{[1]}$ Despite their proven efficacy, several safety concerns such as narrow therapeutic range, drug-drug and drugfood interactions, and bleeding risk restrict warfarin use in NVAF patients. ${ }^{[2-5]}$ Non-VKA oral anticoagulants (NOACs) such as apixaban, dabigatran, and rivaroxaban have recently become available for NVAF patients in UAE. Results from large clinical trials have shown these drugs to be at least as equally effective as warfarin, while being associated with lower risk of bleeding and death from cardiovascular causes. ${ }^{[2,6-8]}$ However, clinical trials are usually conducted on a highly selected group of patients; hence, it may be difficult to generalize the results of these trials to the wider population encountered in daily practice. ${ }^{[9-11]}$ A previous study highlighted key demographic differences between NVAF patients with claims for OACs in UAE and studies conducted in Europe and the USA. ${ }^{[12]}$ Still, there is a lack of real-world evidence regarding OAC use in the Africa-Middle East (AfME) region. Therefore, to address the existing knowledge gap and inform the local and regional healthcare guidelines, the current study utilized the Dubai Real-World Claims Database (DRWD) $)^{[13-15]}$ to understand demographic and clinical characteristics as well as utilization patterns and cost of NVAF patients on apixaban, rivaroxaban, dabigatran, and warfarin in Dubai.

\section{MATERIALS AND METHODS}

\section{Study design}

This was a retrospective cohort analysis using the DRWD. Ethics Committee approval was not required for the analysis of this anonymized retrospective patient dataset. Data on all patients with at least one medical claim with diagnosis of NVAF were extracted from the database. For patients with at least one pharmacy claim for apixaban, dabigatran, rivaroxaban, or warfarin, the index date was defined as the date of the first claim for the index OAC. The analysis population was restricted to adult patients with an index date between January 1, 2015 and March 31, 2019 (index period). Data from the 12 months prior to the index date (pre-index or baseline period) were used to assess patient eligibility for the study and extract information pertaining to demographic characteristics, comorbidities, risk scores, and concomitant medications. Data from the 6-month period between April 1, 2019 and September 30, 2019 (follow-up period) were used to evaluate treatment adherence. The analysis population was divided into four cohorts depending on the index OAC drug. The study phases are represented in Figure 1. Patient inclusion and exclusion criteria are depicted in Figure 2.

The main objectives of the study were to describe the following characteristics of the NVAF patients on OAC

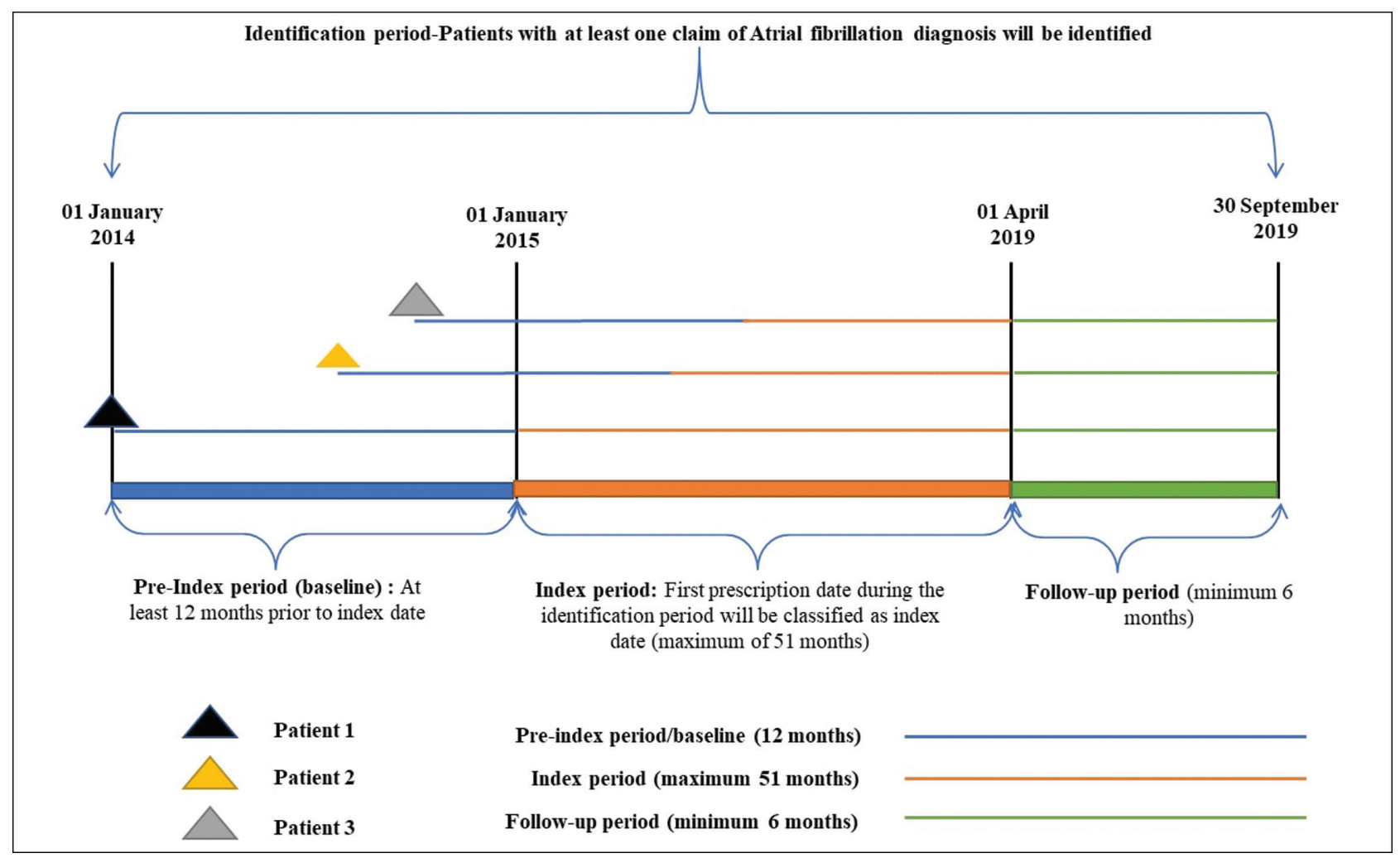

Figure 1: Study phases and timelines. Dubai Real-World Claims Database 


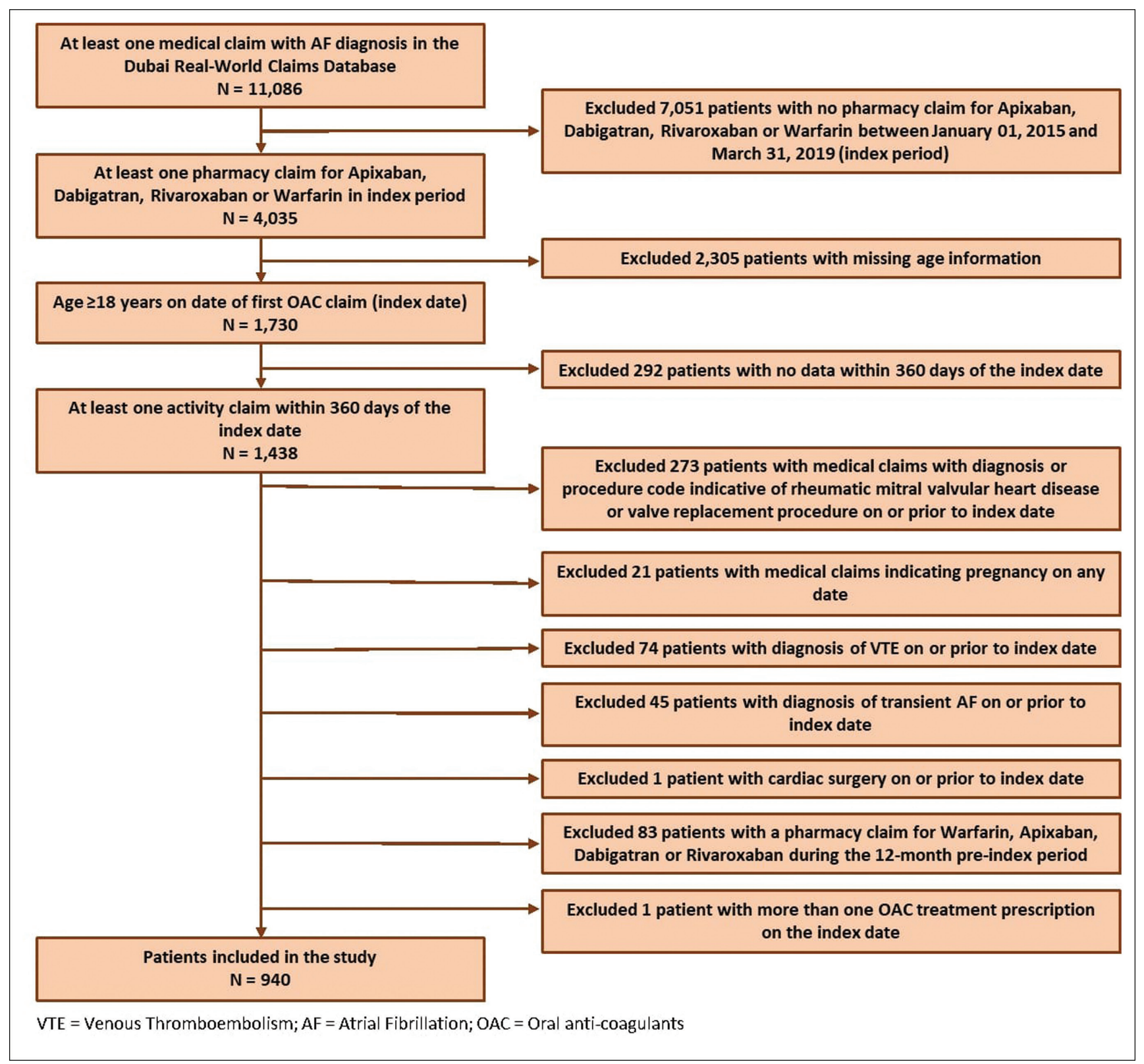

Figure 2: Study flow

treatment (overall and for each of the four OAC cohorts): (1) demographic profile; (2) comorbidities, concomitant medications, and baseline risk; (3) healthcare resource utilization (HCRU) and associated costs; (4) adherence to OACs; and (5) the time to event for outcomes such as stroke, transient ischemic attack (TIA), SE, and major bleeding.

\section{Data sources and variables}

This study utilized data on NVAF patients from DRWD, an anonymized longitudinal patient-level database of insurance claims generated from the private healthcare sector in the Emirate of Dubai. The database contains information from approximately 6.9 million Dubai residents with at least one claim since January 2014. It is a rich source of healthcare data that is valuable for epidemiological studies.
The database contains information on patient demographics, diagnoses, procedures (medical, surgical, and diagnostic), prescriptions, and other related services.

For the current analysis, the following variables were extracted from DRWD:

- Demographics: age and gender

- Clinical characteristics: information pertaining to the following risk scores for prediction of stroke and major bleeding events

1. $\mathrm{CHA}_{2} \mathrm{DS}_{2}$-VASc: age, gender, history of congestive heart failure, hypertension, diabetes mellitus, prior stroke/TIA/SE, and vascular diseases (e.g. myocardial infarction, peripheral arterial disease, aortic plaque $)^{[12]}$ 
2. HAS-BLED: age, hypertension, abnormal renal/liver function, history of stroke, prior major bleeding or predisposition to bleeding, labile international normalized ratio (INR), medication usage predisposing to bleeding (e.g. aspirin, clopidogrel, etc.), and alcohol use $\mathrm{e}^{[16]}$

- Comorbidities: including but not limited to prior stroke, prior bleeding, congestive heart failure (CHF), renal disease, hypertension, and all components of the DeyoCharlson comorbidity index $(\mathrm{CCI})^{[17]}$

- Concomitant drugs

- HCRU and related costs: number of clinic visits, number of hospitalizations and length of stay, number of emergency room visits, number of OAC prescriptions, NOACs dosing pattern along with dose escalation and reduction and costs associated with HCRU for allcauses, major bleeding-related events, and stroke/TIA/ SE-related events

- Time to event of interest: date of first major bleedingrelated event and date of first stroke/TIA/SE-related event

Medical claims were coded using the International Classification of Diseases-Tenth Revision-Clinical Modification (ICD 10-CM), the Current Procedural Terminology (CPT IV), and the Dubai Drug Coding System for Pharmacy claims. ${ }^{[15,18,19]}$

\section{Statistical analysis}

Descriptive analyses of different study parameters were conducted for the overall study population as well as for each of the four OAC cohorts. Continuous variables were described using mean and standard deviation, whereas for categorial variables cumulative and strata-wise counts $(n)$ and percentages (\%) were reported. Treatment adherence was described using medication possession ratio (MPR) and proportion of days covered (PDC), which are widely accepted methods of measuring adherence from claims data. ${ }^{[20,21]}$ These parameters measure treatment adherence in an indirect manner by estimating the proportion of time observed during which patients possess the therapy of interest. In the current analysis, MPR was defined as the proportion of days a patient was on the index OAC during the follow-up period out of the total number of days between the first fill and the last refill plus the number of days' supply in last refill. PDC was defined as the proportion of days supplied for a patient's prescriptions for the index OAC during the follow-up period out of the total number of days the patient was in the follow-up period. A value of $0.8(80 \%)$ or above in these parameters was considered high adherence. ${ }^{[22]}$ Health-related costs extracted from the database were reported in US dollars (USD). All statistical analyses were performed using Statistical Analysis System version 9.4. Due to sample size limitations, formal hypothesis testing between groups was not feasible and hence, comparisons were not conducted.

\section{RESULTS}

A total of 11,086 patients were identified from the DRWD with at least one medical claim of AF diagnosis. Data on 940 patients who had a pharmacy claim for apixaban, dabigatran, rivaroxaban, or warfarin during the index period (January 01, 2015 to March 31, 2019) were included in the analysis. Patient disposition given the study eligibility criteria is presented in Figure 2. The mean age of the overall analysis

\begin{tabular}{|c|c|c|c|c|c|c|c|c|c|c|}
\hline & \multicolumn{2}{|c|}{$\begin{array}{l}\text { Overall cohort } \\
(N=940)\end{array}$} & \multicolumn{2}{|c|}{$\begin{array}{c}\text { Apixaban } \\
(N=328)\end{array}$} & \multicolumn{2}{|c|}{$\begin{array}{c}\text { Dabigatran } \\
(N=\mid 58)\end{array}$} & \multicolumn{2}{|c|}{$\begin{array}{c}\text { Rivaroxaban } \\
(N=338)\end{array}$} & \multicolumn{2}{|c|}{$\begin{array}{l}\text { Warfarin } \\
(N=116)\end{array}$} \\
\hline & $n$ & $\%$ & $n$ & $\%$ & $n$ & $\%$ & $n$ & $\%$ & $n$ & $\%$ \\
\hline \multicolumn{11}{|l|}{ Age } \\
\hline 18 to $<55$ & 357 & $38.0 \%$ & 117 & $35.7 \%$ & 60 & $38.0 \%$ & $13 \mid$ & $38.8 \%$ & 49 & $42.2 \%$ \\
\hline 55 to $<65$ & 257 & $27.3 \%$ & 87 & $26.5 \%$ & 42 & $26.6 \%$ & 99 & $29.3 \%$ & 29 & $25.0 \%$ \\
\hline 65 to $<75$ & 181 & $19.3 \%$ & 64 & $19.5 \%$ & $4 I$ & $25.9 \%$ & 61 & $18.0 \%$ & 15 & $12.9 \%$ \\
\hline$\geq 75$ & 145 & $15.4 \%$ & 60 & $18.3 \%$ & 15 & $9.5 \%$ & 47 & $13.9 \%$ & 23 & $19.8 \%$ \\
\hline Mean age $\pm S D$ & \multicolumn{2}{|c|}{$58.6 \pm 14.7$} & \multicolumn{2}{|c|}{$59.6 \pm 15.3$} & \multicolumn{2}{|c|}{$57.7 \pm 13.7$} & \multicolumn{2}{|c|}{$57.8 \pm 14.8$} & \multicolumn{2}{|c|}{$59.4 \pm 14.4$} \\
\hline \multicolumn{11}{|l|}{ Gender } \\
\hline Male & 693 & $73.7 \%$ & 244 & $74.4 \%$ & 120 & $75.9 \%$ & 249 & $73.7 \%$ & 80 & $69.0 \%$ \\
\hline \multicolumn{11}{|l|}{ Baseline medication use } \\
\hline Antiplatelet drugs & 169 & $18.0 \%$ & 80 & $24.4 \%$ & 17 & $10.8 \%$ & 43 & $12.7 \%$ & 29 & $25.0 \%$ \\
\hline Other anticoagulants & 385 & $41.0 \%$ & 153 & $46.6 \%$ & 49 & $31.0 \%$ & 133 & $39.3 \%$ & 50 & $43.1 \%$ \\
\hline $\begin{array}{l}\text { Angiotensin receptor } \\
\text { blockers }\end{array}$ & 311 & $33.1 \%$ & 115 & $35.1 \%$ & 61 & $38.6 \%$ & 107 & $31.7 \%$ & 28 & $24.1 \%$ \\
\hline Statins & 486 & $51.7 \%$ & 181 & $55.2 \%$ & 78 & $49.4 \%$ & 164 & $48.5 \%$ & 63 & $54.3 \%$ \\
\hline Aspirin & 321 & $34.1 \%$ & 125 & $38.1 \%$ & 43 & $27.2 \%$ & 107 & $31.7 \%$ & 46 & $39.7 \%$ \\
\hline
\end{tabular}


cohort was $58.6 \pm 14.7$ years, with males making up $73.7 \%$ of the analysis population [Table 1]. The most commonly used concomitant medications at baseline were statins $(n=486$, $51.7 \%)$, followed by other anticoagulants (mainly heparin) $(n=385,41.0 \%)$ and aspirin $(n=321,34.1 \%)$. Among patients without an OAC claim who met all other inclusion criteria and none of the exclusion criteria [Figure 2], 435 patients with $\mathrm{CHA}_{2} \mathrm{DS}_{2}$-VASc score $\geq 2$ were on antiplatelets (aspirin, clopidogrel) only (analysis not shown).

The baseline risks for stroke and major bleeding as well as the comorbidity burden for the patients in the study are depicted in Table 2. The majority of patients had a $\mathrm{CHA}_{2} \mathrm{DS}_{2}-\mathrm{VASc}$ score $\geq 2(n=616,65.5 \%)$, placing this cohort at a high risk of stroke or SE. The proportion of high-risk patients was higher in the warfarin cohort $(74.1 \%)$ than in all the NOACs cohorts (ranging from $60.4 \%$ among rivaroxaban patients to $68.3 \%$ in the apixaban group); however, the mean $\mathrm{CHA}_{2} \mathrm{DS}_{2}$-VASc score was comparable in the warfarin (2.7) and apixaban (2.6) cohorts. About $12.8 \%$ of the overall OAC cohort had a $\mathrm{CHA}_{2} \mathrm{DS}_{2}$-VASc score of 0 . HAS-BLED scores could not be calculated in this study since alcohol use and INR results were not captured in DRWD. From the individual components of the HAS-BLED score available in the dataset, abnormal renal or liver function, bleeding, and drug use were more prevalent in the warfarin cohort than in the NOACs cohorts, whereas hypertension and history of stroke were more frequent in the dabigatran group. Rivaroxaban users had the lower percentages of patients with hypertension, history of stroke, elderly age ( $>65$ years), and drug use. The mean CCI score at baseline for the overall population was $1.6 \pm 1.8$, with the warfarin cohort having the highest CCI score (2.2) and the dabigatran cohort having the lowest (1.2) mean. Overall, a third of the patients had a CCI score of $0(n=316,33.6 \%)$, indicating no significant comorbidities apart from AF, with the majority of the cohort having a CCI score of $1-2(n=406,43.2 \%)$ and less than a quarter with a CCI score $\geq 3(n=218,23.2 \%)$.

Table 3 presents the index OAC dose and subsequent dose changes in the four OAC cohorts. Half of the patients in the dabigatran cohort started with an index dose of $110 \mathrm{mg}$ twice daily $(n=79 ; 50.0 \%)$, whereas most of the other half $(n=66 ; 41.8 \%)$ started on a standard dose of $150 \mathrm{mg}$ twice daily and less than $10 \%$ started on a different dose $(n=13$; $8.2 \%)$. The prescriptions for rivaroxaban and apixaban were

\begin{tabular}{|c|c|c|c|c|c|c|c|c|c|c|}
\hline & \multicolumn{2}{|c|}{$\begin{array}{l}\text { Overall cohort } \\
\qquad(N=940)\end{array}$} & \multicolumn{2}{|c|}{$\begin{array}{c}\text { Apixaban } \\
(N=328)\end{array}$} & \multicolumn{2}{|c|}{$\begin{array}{c}\text { Dabigatran } \\
(N=158)\end{array}$} & \multicolumn{2}{|c|}{$\begin{array}{l}\text { Rivaroxaban } \\
(N=338)\end{array}$} & \multicolumn{2}{|c|}{$\begin{array}{l}\text { Warfarin } \\
(N=1 \mid 6)\end{array}$} \\
\hline & $n$ & $\%$ & $n$ & $\%$ & $n$ & $\%$ & $n$ & $\%$ & $n$ & $\%$ \\
\hline \multicolumn{11}{|c|}{$\mathrm{CHA}_{2} \mathrm{DS}_{2}$-VASc risk score category } \\
\hline 0 & 120 & $12.8 \%$ & $4 \mid$ & $12.5 \%$ & 22 & $13.9 \%$ & 49 & $14.5 \%$ & 8 & $6.9 \%$ \\
\hline I & 204 & $21.7 \%$ & 63 & $19.2 \%$ & 34 & $21.5 \%$ & 85 & $25.1 \%$ & 22 & $19.0 \%$ \\
\hline $2+$ & 616 & $65.5 \%$ & 224 & $68.3 \%$ & 102 & $64.6 \%$ & 204 & $60.4 \%$ & 86 & $74.1 \%$ \\
\hline Mean \pm SD & \multicolumn{2}{|c|}{$2.4 \pm 1.7$} & \multicolumn{2}{|c|}{$2.6 \pm 1.8$} & \multicolumn{2}{|c|}{$2.3 \pm 1.6$} & \multicolumn{2}{|c|}{$2.2 \pm 1.6$} & \multicolumn{2}{|c|}{$2.7 \pm 1.7$} \\
\hline \multicolumn{11}{|c|}{$\mathrm{CHA}_{2} \mathrm{DS}_{2}$-VASc score components } \\
\hline Congestive heart failure & $|5|$ & $16.1 \%$ & 69 & $21.0 \%$ & 20 & $12.7 \%$ & 36 & $10.7 \%$ & 26 & $22.4 \%$ \\
\hline Hypertension & 694 & $73.8 \%$ & 250 & $76.2 \%$ & 123 & $77.8 \%$ & 236 & $69.8 \%$ & 85 & $73.3 \%$ \\
\hline Age $65-74$ at index date & 181 & $19.3 \%$ & 64 & $19.5 \%$ & 41 & $25.9 \%$ & 61 & $18.0 \%$ & 15 & $12.9 \%$ \\
\hline Age $\geq 75$ at index date & 145 & $15.4 \%$ & 60 & $18.3 \%$ & 15 & $9.5 \%$ & 47 & $13.9 \%$ & 23 & $19.8 \%$ \\
\hline Diabetes mellitus & 369 & $39.3 \%$ & 140 & $42.7 \%$ & 55 & $34.8 \%$ & 117 & $34.6 \%$ & 57 & $49.1 \%$ \\
\hline Stroke/TIA/SE & 85 & $9.0 \%$ & 33 & $10.1 \%$ & 18 & $1 \mathrm{I} .4 \%$ & 24 & $7.1 \%$ & 10 & $8.6 \%$ \\
\hline Vascular disease & |5| & $16.1 \%$ & 51 & $15.5 \%$ & 24 & $15.2 \%$ & 51 & $15.1 \%$ & 25 & $21.6 \%$ \\
\hline Female & 247 & $26.3 \%$ & 84 & $25.6 \%$ & 38 & $24.1 \%$ & 89 & $26.3 \%$ & 36 & $31.0 \%$ \\
\hline \multicolumn{11}{|c|}{ HAS-BLED score components present in the dataset } \\
\hline Hypertension & 694 & $73.8 \%$ & 250 & $76.2 \%$ & 123 & $77.8 \%$ & 236 & $69.8 \%$ & 85 & $73.3 \%$ \\
\hline Abnormal renal/liver function & 171 & $18.2 \%$ & 65 & $19.8 \%$ & 23 & $14.6 \%$ & 53 & $15.7 \%$ & 30 & $25.9 \%$ \\
\hline Stroke & 78 & $8.3 \%$ & 30 & $9.1 \%$ & 16 & $10.1 \%$ & 22 & $6.5 \%$ & 10 & $8.6 \%$ \\
\hline Bleeding & 192 & $20.4 \%$ & 68 & $20.7 \%$ & 22 & $13.9 \%$ & 61 & $18.0 \%$ & 41 & $35.3 \%$ \\
\hline Age $\geq 65$ at index date & 326 & $34.7 \%$ & 124 & $37.8 \%$ & 56 & $35.4 \%$ & 108 & $32.0 \%$ & 38 & $32.8 \%$ \\
\hline Drug use & 470 & $50.0 \%$ & 176 & $53.7 \%$ & 74 & $46.8 \%$ & 155 & $45.9 \%$ & 65 & $56.0 \%$ \\
\hline \multicolumn{11}{|l|}{$\mathrm{CCl}$ score } \\
\hline 0 & 316 & $33.6 \%$ & 93 & $28.4 \%$ & 63 & $39.9 \%$ & 139 & $4 I .1 \%$ & 21 & $18.1 \%$ \\
\hline $\mathrm{I}-2$ & 406 & $43.2 \%$ & 139 & $42.4 \%$ & 69 & $43.7 \%$ & 144 & $42.6 \%$ & 54 & $46.6 \%$ \\
\hline$\geq 3$ & 218 & $23.2 \%$ & 96 & $29.3 \%$ & 26 & $16.5 \%$ & 55 & $16.3 \%$ & 41 & $35.3 \%$ \\
\hline Mean $\pm S D$ & \multicolumn{2}{|c|}{$1.6 \pm 1.8$} & \multicolumn{2}{|c|}{$1.9 \pm 2.0$} & \multicolumn{2}{|c|}{$1.2 \pm 1.5$} & \multicolumn{2}{|c|}{$1.3 \pm 1.6$} & \multicolumn{2}{|c|}{$2.2 \pm 1.9$} \\
\hline
\end{tabular}

$\mathrm{CHA}_{2} \mathrm{DS}_{2}-\mathrm{VASc}$ : Congestive heart failure, hypertension, age, diabetes, previous stroke/TIA/SE, vascular disease, sex; HAS-BLED: hypertension, abnormal renal/liver function and stroke, bleeding, labile INR, elderly age and prior alcohol/drug usage/medication usage; CCI: Deyo-Charlson comorbidity index; TIA: transient ischemic attack; SE: systemic embolism; SD: standard deviation.

Individual HAS-BLED risk scores could not be calculated given that information for labile international normalized ratio and alcohol use was not available in the dataset. 
predominantly skewed toward standard doses, with over $70 \%$ of the patients in both cohorts being initially prescribed a dose of $5 \mathrm{mg}$ twice daily for apixaban and $20 \mathrm{mg}$ once daily for rivaroxaban. Warfarin had variable dosing (i.e. there were no clear dose preferences), and a standard index dose could not be defined.

From the baseline cohort of 940 patients, only 622 (66.2\%) patients had data during the 180-day follow-up period, and among them, $436(70.1 \%)$ patients had two or more prescriptions in the database. When comparing prescriptions for those with available data, the majority of patients $(n=347$; $79.6 \%$ ) did not change dose during the follow-up period, with the proportion of patients having at least one dose modification being below $16 \%$ for three NOACs drug cohorts but reaching $64 \%$ in the warfarin group. Among those who changed doses, 49 (11.2\%) patients had a dose reduction, which was more frequently observed in the warfarin cohort compared with the NOACs groups [Table 3].

At 180 days of follow-up, high MPR and PDC $(\geq 80 \%)$ were achieved in $86.8 \%$ and $43.1 \%$ of the patients, respectively [Table 4]. Patients on warfarin had the greatest percentage of high MPR (89.2\%) followed by apixaban (88.2\%), dabigatran (86.5\%), and rivaroxaban (85.0\%). The overall mean MPR was $161 \%$ mainly driven by warfarin users for which the mean MPR was five times higher than for the three NAOC cohorts. In contrast, the percentage of patients achieving high PDC was similar across cohorts, with dabigatran users having the highest figure (54.1\%) followed closely by the warfarin (51.4\%) and apixaban (50.2\%) cohorts. In the overall cohort, the mean PDC was $62 \%$. During the follow-

\begin{tabular}{|c|c|c|c|c|c|c|c|c|c|c|}
\hline & & & \multicolumn{2}{|c|}{$\begin{array}{c}\text { Apixaban } \\
(N=328)\end{array}$} & \multicolumn{2}{|c|}{$\begin{array}{c}\text { Dabigatran } \\
(N=\mid 58)\end{array}$} & \multicolumn{2}{|c|}{$\begin{array}{c}\text { Rivaroxaban } \\
(N=338)\end{array}$} & & \\
\hline & & & $n$ & $\%$ & $\bar{n}$ & $\%$ & $n$ & $\%$ & & \\
\hline \multicolumn{11}{|l|}{ Index dose } \\
\hline Standard dose & & & 233 & $71.0 \%$ & 66 & $41.8 \%$ & 237 & $70.1 \%$ & & \\
\hline Lower dose & & & 95 & $29.0 \%$ & 79 & $50.0 \%$ & 75 & $22.2 \%$ & & \\
\hline \multirow[t]{3}{*}{ All others } & & & 0 & $0.0 \%$ & 13 & $8.2 \%$ & 26 & $7.7 \%$ & & \\
\hline & $\begin{array}{l}\text { Follo } \\
(N=6\end{array}$ & ohort & Apix & $V=203)$ & $\begin{array}{l}\mathrm{Dat} \\
(\mathrm{N}=\end{array}$ & & $\begin{array}{l}\text { Riva } \\
(N=2\end{array}$ & & Wa & $V=74)$ \\
\hline & $n$ & $\%$ & $n$ & $\%$ & $n$ & $\%$ & $n$ & $\%$ & $\mathrm{n}$ & $\%$ \\
\hline \multicolumn{11}{|c|}{ Number of prescriptions during follow-up } \\
\hline 1 & 186 & $29.9 \%$ & 58 & $28.6 \%$ & 35 & $31.5 \%$ & 72 & $30.8 \%$ & 21 & $28.4 \%$ \\
\hline$\geq 2$ & 436 & $70.1 \%$ & 145 & $71.4 \%$ & 76 & $68.5 \%$ & 162 & $69.2 \%$ & 53 & $71.6 \%$ \\
\hline \multicolumn{11}{|c|}{ Dose change during follow-up for patients $\geq 2$ prescriptions in the 180 -day period $(N=436)$} \\
\hline No change & 347 & $79.6 \%$ & 122 & $84.1 \%$ & 67 & $88.2 \%$ & 139 & $85.8 \%$ & 19 & $35.8 \%$ \\
\hline Dose increase* & 40 & $9.2 \%$ & 11 & $7.6 \%$ & 5 & $6.6 \%$ & 10 & $6.2 \%$ & 14 & $26.4 \%$ \\
\hline Dose decrease* & 49 & $11.2 \%$ & 12 & $8.3 \%$ & 4 & $5.3 \%$ & 13 & $8.0 \%$ & 20 & $37.7 \%$ \\
\hline
\end{tabular}

\begin{tabular}{|c|c|c|c|c|c|c|c|c|c|c|}
\hline & \multicolumn{2}{|c|}{$\begin{array}{c}\text { Follow-up } \\
\text { cohort }(N=622)\end{array}$} & \multicolumn{2}{|c|}{$\begin{array}{l}\text { Apixaban } \\
(N=203)\end{array}$} & \multicolumn{2}{|c|}{$\begin{array}{c}\text { Dabigatran } \\
(N=|| I)\end{array}$} & \multicolumn{2}{|c|}{$\begin{array}{c}\text { Rivaroxaban } \\
(N=234)\end{array}$} & \multicolumn{2}{|c|}{$\begin{array}{l}\text { Warfarin } \\
(N=74)\end{array}$} \\
\hline & $n$ & $\%$ & $n$ & $\%$ & $n$ & $\%$ & $n$ & $\%$ & $n$ & $\%$ \\
\hline \multicolumn{11}{|c|}{ Medication possession ratio (MPR) } \\
\hline$<80 \%$ & 82 & $13.2 \%$ & 24 & $11.8 \%$ & 15 & $13.5 \%$ & 35 & $15.0 \%$ & 8 & $10.8 \%$ \\
\hline$\geq 80 \%$ & 540 & $86.8 \%$ & 179 & $88.2 \%$ & 96 & $86.5 \%$ & 199 & $85.0 \%$ & 66 & $89.2 \%$ \\
\hline \multicolumn{11}{|c|}{ Proportion of days covered (PDC) } \\
\hline$<80 \%$ & 354 & $56.9 \%$ & 101 & $49.8 \%$ & 51 & $45.9 \%$ & 166 & $70.9 \%$ & 36 & $48.6 \%$ \\
\hline$\geq 80 \%$ & 268 & $43.1 \%$ & 102 & $50.2 \%$ & 60 & $54.1 \%$ & 68 & $29.1 \%$ & 38 & $51.4 \%$ \\
\hline \multicolumn{11}{|c|}{ Patients switching to a different $O A C$} \\
\hline Anticoagulant switch & 74 & $11.9 \%$ & 13 & $6.4 \%$ & 23 & $20.7 \%$ & 23 & $9.8 \%$ & 15 & $20.3 \%$ \\
\hline Switched to apixaban & 36 & $48.6 \%$ & - & - & 14 & $60.9 \%$ & 17 & $73.9 \%$ & 5 & $33.3 \%$ \\
\hline Switched to dabigatran & 9 & $12.2 \%$ & 2 & $15.4 \%$ & - & - & 4 & $17.4 \%$ & 3 & $20.0 \%$ \\
\hline Switched to rivaroxaban & 25 & $33.8 \%$ & 10 & $76.9 \%$ & 8 & $34.8 \%$ & - & - & 7 & $46.7 \%$ \\
\hline Switched to warfarin & 4 & $5.4 \%$ & I & $7.7 \%$ & I & $4.3 \%$ & 2 & $8.7 \%$ & - & - \\
\hline
\end{tabular}


up period, 74 patients (11.9\%) switched to a different OAC, with the highest percentages observed for the dabigatran (20.7\%) and warfarin (20.3\%) cohorts. Among the switchers, almost half of the patients (48.6\%) switched to apixaban, a third (33.8\%) switched to rivaroxaban, $12.2 \%$ switched to dabigatran, and only $5.4 \%$ switched to warfarin.

The mean number of all-cause HCRU claims during the 180 days follow-up period was 18.5 , and the associated cost was 9747 USD [Table 5]. Warfarin users accounted for both the highest number of claims and highest cost for all-cause HCRU (claims: 26.0 and cost: 14,697 USD), whereas apixaban users had the lowest number of claims and lowest cost for all-cause HCRU (claims: 17.3 and cost: 7774 USD). The mean numbers of all-cause claims for the NOAC cohorts were largely similar (apixaban: 17.3, dabigatran: 17.9, rivaroxaban: 17.6), and their costs were much lower than the warfarin cohort claims. Warfarin users also had a much higher mean claims and cost for two major outcome events-major bleeding (claims: 0.8 and cost: 3539 USD) and stroke/TIA/SE (0.8 and 3446 USD) — compared with NOAC users. Warfarin users also reported greater lengths of hospital stay compared with NOAC users for major bleeding and stroke/TIA/SE.

Time to first event analyses using all available follow-up estimated the shortest mean time to major bleeding for the warfarin cohort and the shortest time to stroke/TIA/ SE for the dabigatran group [Table 6]. Interestingly, for the overall cohort, the mean number of days to a first major bleeding-related event $(304.7 \pm 351.3$ days $)$ was greater than the mean number of days to a first event of stroke/TIA/SE (183.0 \pm 244.0 days). A similar pattern was observed for the four cohorts.

\section{DISCUSSION}

This claims database study explored the demographic profile, clinical characteristics, utilization patterns, and health-related costs of NVAF patients who had pharmacy claims for apixaban, dabigatran, rivaroxaban, or warfarin. To the best of our knowledge, the current analysis is not only the largest study utilizing DRWD but also the largest to describe the clinical characteristics and HCRU pertaining to OAC use among NVAF patients in Dubai. The study findings address the knowledge gap related to HCRU and utilization patterns of VKA and NOACs, complement previous efforts to characterize NVAF patients with claims for oral anticoagulants in the $\mathrm{UAE},{ }^{[12]}$ and provide insights on adherence rates not only in Dubai but also across the UAE.

The mean age of the overall sample was below 60 years, with no significant difference across the different drug cohorts. This is similar to a prior large study on AF patients in the Gulf region. ${ }^{[23]}$ The findings of this study revealed that more than a third of NVAF patients-overall and in the four individual drug cohorts-were below 55 years age. This is somewhat unexpected as NVAF is commonly associated with older age, and published literature from western countries suggests that NVAF increases exponentially with increasing age. ${ }^{[24]}$ Compared to the aforementioned study in the Gulf region by Zubaid et al., the gender distribution in the current study was predominantly skewed toward males ( $74 \%$ males). This could be attributed to male preponderance in DRWD rather than difference in NVAF risk across genders.

Interestingly, among the NVAF patients excluded from the analysis because they were not prescribed oral anticoagulants, there were 435 patients at high-risk for stroke who were only treated with antiplatelet therapy. Similarly, about $12 \%$ of the patients with $\mathrm{CHA}_{2} \mathrm{DS}_{2}$-VASc score of 0 were treated with OACs, none of these patients had other compelling indications for anticoagulation-like LV thrombus, LA thrombus, or hypercoagulable state (protein $\mathrm{S}$ or protein $\mathrm{C}$ deficiencies) prior or on index date. These findings may highlight an opportunity for patient and physician education in the management of NVAF in UAE.

\begin{tabular}{|c|c|c|c|c|c|}
\hline & $\begin{array}{l}\text { Follow-up cohort } \\
\quad(N=622)\end{array}$ & $\begin{array}{l}\text { Apixaban } \\
(N=203)\end{array}$ & $\begin{array}{c}\text { Dabigatran } \\
(\mathrm{N}=\mathrm{I} \text { III) }\end{array}$ & $\begin{array}{c}\text { Rivaroxaban } \\
(N=234)\end{array}$ & $\begin{array}{l}\text { Warfarin } \\
(N=74)\end{array}$ \\
\hline \multicolumn{6}{|l|}{ All-cause healthcare utilization } \\
\hline Mean number of claims \pm SD & $18.5 \pm 19.2$ & $17.3 \pm 16.5$ & $17.9 \pm 14.9$ & $17.6 \pm 15.8$ & $26 \pm 34.6$ \\
\hline Mean gross cost USD \pm SD & $9,747 \pm 19,122$ & $7,774 \pm 9,766$ & $8,948 \pm 12,555$ & $10,273 \pm 23,045$ & $14,697 \pm 29,607$ \\
\hline \multicolumn{6}{|c|}{ Major bleeding-related healthcare utilization } \\
\hline Mean number of claims \pm SD & $0.3 \pm 1.1$ & $0.1 \pm 0.7$ & $0.2 \pm 0.9$ & $0.2 \pm 1.0$ & $0.8 \pm 2.2$ \\
\hline Mean gross cost USD \pm SD & $683 \pm 8,223$ & $435 \pm 3,609$ & $446 \pm 3,974$ & $108 \pm 840$ & $3,539 \pm 22,440$ \\
\hline Mean length of stay (days) \pm SD & $0.5 \pm 5.2$ & $0.2 \pm 1.9$ & $0.4 \pm 4.6$ & $0.1 \pm 0.8$ & $2.5 \pm 13.5$ \\
\hline \multicolumn{6}{|c|}{ Stroke/TIA/SE-related healthcare utilization } \\
\hline Mean number of claims \pm SD & $0.3 \pm 1.9$ & $0.4 \pm 1.7$ & $0.3 \pm 1.2$ & $0.2 \pm 0.9$ & $0.8 \pm 4.0$ \\
\hline Mean gross cost USD \pm SD & $745 \pm 8,535$ & $247 \pm 1,344$ & $24 I \pm 1,369$ & $562 \pm 5,601$ & $3,446 \pm 22,431$ \\
\hline Mean length of stay (days) $\pm S D$ & $1.0 \pm 10.5$ & $0.3 \pm 1.7$ & $0.1 \pm 0.7$ & $1.3 \pm 15.0$ & $2.9 \pm 14.2$ \\
\hline
\end{tabular}


Multimorbidity has been recognized as an independent risk factor for $\mathrm{AF}^{[25]}$ In this study, the overall baseline CCI score for NVAF patients that started OAC treatment was above 1.5 , with the largest comorbidity burden observed for the warfarin cohort. This is not entirely unexpected as warfarin is more commonly prescribed for NVAF patients with (or at greater risk of) cardiovascular and cerebrovascular ailments, which is corroborated in this study by a positive history of $\mathrm{CHF}$ and cerebrovascular diseases in more than $20 \%$ and $10 \%$, respectively, in the warfarin cohort. Hypertension and diabetes mellitus (with and without complications) were the most common comorbidities reported, which was also observed in a previous study on heart failure patients in Dubai. ${ }^{[26]}$

The $\mathrm{CHA}_{2} \mathrm{DS}_{2}$-VASc score is a widely accepted tool for quantifying the risk of stroke among all types of AF patients. ${ }^{[12,27]}$ In the current study, mean $\mathrm{CHA}_{2} \mathrm{DS}_{2}$-VASc score for the overall cohort was 2.4, which is much lower than that reported from a pooled analysis based on registry data from multiple countries in Asia, Europe, and Latin America. ${ }^{[28]}$ This could suggest a lesser risk of stroke among NVAF patients in DRWD compared with other countries. However, more plausible explanations include that DRWD may not be entirely representative of the NVAF patient population in Dubai and could have higher representation of patients with lesser risk of stroke, or that there was underreporting of the risk factors used to calculate the score. Another important finding is that the warfarin cohort had higher baseline $\mathrm{CHA}_{2} \mathrm{DS}_{2}$-VASc scores compared with rest of the cohorts, as well as higher prevalence of components of the HAS-BLED score related to abnormal renal/liver function, bleeding, and drug use. As previously discussed, warfarin tends to be the OAC of choice for NVAF patients with comorbidities, resulting in higher (poorer) scores in each of the scales compared with the NOAC cohorts.

All NOACs were commonly prescribed in standard doses, which is consistent with a population-based cross-sectional study on NVAF patients in the UK. ${ }^{[29]}$ Reduced NOAC dose (unless indicated) may have important clinical implications as the intended protective benefits may not be obtained. There was no clear predilection toward higher or lower dosage of warfarin possibly owing to the fact that warfarin doses are often decided on the basis of a delicate balance between reduction of stroke risk and potential side effects. ${ }^{[30]}$ While only $10-16 \%$ of the patients on NOAC had changes in drug dose during the follow-up period, only a third of warfarin users remained on the same dose during the 180day follow-up. This suggests that patients on warfarin require more frequent dose titration compared with NOAC users.

Adherence rates were high for all OAC cohorts, with excellent levels of MPR and acceptable levels of PDC overall. These adherence rates are better than those reported for most drugs prescribed for chronic cardiovascular ailments, but are similar to adherence rates of OACs among NVAF patients across the globe. ${ }^{[31]}$ During follow-up, the apixaban and rivaroxaban cohorts had the lowest percentage of switchers to a different anticoagulant, which was consistent with other studies showing that dabigatran has the highest switch rate among NOAC users. ${ }^{[32,33]}$ Interestingly, the switching percentage in the dabigatran and warfarin cohorts were very similar at $20-21 \%$.

The warfarin cohort not only reported the highest number of all-cause HCRU claims but also accounted for the highest cost among all cohorts. Higher costs could be a consequence of increased length of stay for major bleeding and stroke-related hospitalization (length of stay was at a minimum two times higher in the warfarin cohort than in any of the NOAC cohorts). The higher prevalence of preexisting comorbidities in warfarin users (as indicated by CCI scores) compared with the NOAC cohorts could be another potential explanation for the increased HCRU cost and length of stay. Among the NOAC cohorts, there was not much difference in terms of all-cause HCRU claims, but cost was higher for rivaroxaban users. This could be attributed to longer length of stroke-related hospitalization stay in the rivaroxaban group, which is consistent with a study on US patients who switched from warfarin to NOACs. ${ }^{[34]}$ Apixaban users had the lowest number of claims and the

\begin{tabular}{|c|c|c|c|c|c|}
\hline & $\begin{array}{l}\text { Overall cohort } \\
\quad(n=622)\end{array}$ & $\begin{array}{l}\text { Apixaban cohort } \\
(n=203)\end{array}$ & $\begin{array}{l}\text { Dabigatran cohort } \\
\qquad(n=I I I)\end{array}$ & $\begin{array}{l}\text { Rivaroxaban cohort } \\
(n=234)\end{array}$ & $\begin{array}{l}\text { Warfarin cohort } \\
(n=74)\end{array}$ \\
\hline \multicolumn{6}{|c|}{ Time to first major bleeding-related event (days) } \\
\hline$N$ & 110 & 28 & 16 & 44 & 22 \\
\hline Mean days \pm SD & $304.7 \pm 351.3$ & $239.9 \pm 266.9$ & $321.2 \pm 412.4$ & $379.0 \pm 403.1$ & $226.6 \pm 266.6$ \\
\hline \multicolumn{6}{|c|}{ Time to first stroke/TIA/SE-related event (days) } \\
\hline$N$ & 79 & 23 & 16 & 26 & 14 \\
\hline Mean days \pm SD & $183.0 \pm 244.0$ & $136.5 \pm 226.1$ & $125.6 \pm 183.3$ & $261.1 \pm 318.8$ & $179.8 \pm 127.8$ \\
\hline
\end{tabular}


lowest cost for all-cause HCRU. Interestingly, the HCRU claims and costs related to major bleeding and stroke/SE accounted for only a small fraction of the overall HCRU claims/costs; this could be indirect evidence of the beneficial effects of OACs among NVAF patients and could point to an increased beneficial effect for NOACs compared with warfarin as judged by the significantly smaller fraction of the overall HCRU related to the two relevant event types in the former subgroup.

Assuming that longer time to event indicates lower risk, rivaroxaban users had lower risk of stroke/SE compared with other OACs users. This may be explained by the fact that rivaroxaban users had lower $\mathrm{CHA}_{2} \mathrm{DS}_{2}$-VASc score and some components of HAS-BLED score. Surprisingly, warfarin users had a shorter interval to first major bleeding event compared with NOAC users. This is similar to the findings from the NOAC trials but contradicts a claims database study on NVAF patients in the USA. ${ }^{[8,35,36]}$ Further research is needed to understand whether these inconsistent findings resulted from the inherent differences between trials and actual clinical usage (real-world data) or because of some characteristic variations between warfarin and NOAC users in DRWD.

As with any retrospective analysis of claims data, there are a few limitations that merit discussion. First, the study parameters may have been affected by measurement error. The study cohorts were defined based on claims for filled prescriptions made by the patients. However, this may not always indicate that the patients actually consumed the drug (or consumed it as prescribed) which would result in exposure misclassification. Similarly, exposure misclassification would also result if the patients obtained OAC drugs using out-of-pocket expenditure (i.e. without filing a claim). As with the drugs, the information regarding the outcome of interest (diagnosis code on a medical claim) could also be inaccurate and, in turn, lead to outcome misclassification. Measurement error, if present, may reduce the internal validity of the study. ${ }^{[37]}$ However, there are no reasons to believe that the extent and the impact of the aforementioned bias in the current study are more than other claims database studies conducted across the globe. Secondly, there could be issues regarding external validity/ generalizability of real-world data studies as they may not be representative of the whole population. Nevertheless, with DRWD being the largest source of secondary data on NVAF patients in Dubai, the findings may still be generalizable to a large population. Further, besides errors in collecting/coding of available parameters, claims database studies are often limited by lack/insufficiencies of important clinical data. ${ }^{[37]}$ For example, in the current study, INR measurement-an important parameter for determining the dose titration of OACs-was unavailable. Therefore, the time spent in therapeutic range for VKA cohort could not be calculated. Also, the medical and prescription histories were limited to claims made during study period. This may have resulted in incomplete information pertaining to comorbidities and concomitant medications for some patients. Finally, while the overall and cohort-specific sample sizes for this study were quite reasonable, data were scarce for some of the subgroup analyses. For example, the data available for some of the strata for medication adherence and drug switching calculation were very limited. Caution should be exercised while interpreting such findings.

Despite the limitations, this descriptive real-world study provides valuable insights about NVAF patients in Dubai and is expected to aid clinical decision-making regarding OACs use in UAE. While most of the results from the current study are consistent with the available literature, some findings deviate from prior studies. Carefully designed future researches, preferably based on primary data, may overcome the limitations of the current study and allow for further exploration of the discordant findings.

\section{Acknowledgements}

We thank Sri Harshadeep Chilukuri for his assistance during data analysis. Medical writing support was provided by Aritra Das and Eleonora Staines Urias at IQVIA and was funded by Pfizer.

\section{Financial support and sponsorship}

This research was sponsored by Pfizer Gulf FZ LLC.

\section{Conflicts of interest}

Ahmed Ghorab and Jean Joury are paid employees of Pfizer Gulf, with ownership of stocks in Pfizer Inc. Nancy Awad, Badarinath Chickballapur Ramachandrachar, and Ashok Natarajan are employees of IQVIA who were paid consultants to Pfizer in connection with the development of this manuscript. Mohamed Farghaly and Moutaz El Kadri have no conflicts to declare.

\section{RefERENCES}

1. January CT, Wann LS, Calkins H, Chen LY, Cigarroa JE, Cleveland JC Jr, et al. 2019 AHA/ACC/HRS Focused Update of the 2014 AHA/ACC/HRS Guideline for the Management of Patients with Atrial Fibrillation: A Report of the American College of Cardiology/American Heart Association Task Force on Clinical Practice Guidelines and the Heart Rhythm Society in collaboration with the Society of Thoracic Surgeons. Circulation 2019;140:e125-51.

2. Patel MR, Mahaffey KW, Garg J, Pan G, Singer DE, Hacke W, et al.; ROCKET AF Investigators. Rivaroxaban versus warfarin in nonvalvular atrial fibrillation. N Engl J Med 2011;365:883-91. 
3. Agarwal S, Hachamovitch R, Menon V. Current trial-associated outcomes with warfarin in prevention of stroke in patients with nonvalvular atrial fibrillation: A meta-analysis. Arch Intern Med 2012;172:623-31; discussion 631-3.

4. Sulzgruber P, Wassmann S, Semb AG, Doehner W, Widimsky P, Gremmel T, et al. Oral anticoagulation in patients with non-valvular atrial fibrillation and a CHA2DS2-VASc score of 1: A current opinion of the European Society of Cardiology Working Group on Cardiovascular Pharmacotherapy and European Society of Cardiology Council on Stroke. Eur Heart J Cardiovasc Pharmacother 2019;5:171-80.

5. Tomaselli GF, Mahaffey KW, Cuker A, Dobesh PP, Doherty JU, Eikelboom JW, et al. 2020 ACC Expert Consensus Decision Pathway on management of bleeding in patients on oral anticoagulants: A report of the American College of Cardiology Solution Set Oversight Committee. J Am Coll Cardiol 2020;76:594-622.

6. Giugliano RP, Ruff CT, Braunwald E, Murphy SA, Wiviott SD, Halperin JL, et al.; ENGAGE AF-TIMI 48 Investigators. Edoxaban versus warfarin in patients with atrial fibrillation. N Engl J Med 2013;369:2093-104.

7. Granger CB, Alexander JH, McMurray JJ, Lopes RD, Hylek EM, Hanna M, et al.; ARISTOTLE Committees and Investigators. Apixaban versus warfarin in patients with atrial fibrillation. N Engl J Med 2011;365:981-92.

8. Connolly SJ, Ezekowitz MD, Yusuf S, Eikelboom J, Oldgren J, Parekh A, et al.; RE-LY Steering Committee and Investigators. Dabigatran versus warfarin in patients with atrial fibrillation. N Engl J Med 2009;361:1139-51.

9. May GS, DeMets DL, Friedman LM, Furberg C, Passamani E. The randomized clinical trial: Bias in analysis. Circulation 1981;64:669-73.

10. Viera AJ, Bangdiwala SI. Eliminating bias in randomized controlled trials: Importance of allocation concealment and masking. Fam Med 2007;39:132-7.

11. Kim HS, Lee S, Kim JH. Real-world evidence versus randomized controlled trial: Clinical research based on electronic medical records. J Korean Med Sci 2018;33:e213.

12. Lip GY, Nieuwlaat R, Pisters R, Lane DA, Crijns HJ. Refining clinical risk stratification for predicting stroke and thromboembolism in atrial fibrillation using a novel risk factor-based approach: The Euro Heart Survey on Atrial Fibrillation. Chest 2010;137:263-72.

13. Mahagaonkar SB. Drug safety assessment using Dubai Real World Claims Database (DRWD), in Joint International Conference and Expo on Industrial Pharmacy \& 5th Global Pharmacovigilance Summit. J Develop Drugs 2016:April 28-29, 2016. Dubai, UAE.

14. El Kadri M, Bazargani N, Farghaly M, Mohamed R, Awad N, Natarajan A, et al. Profiling clinical characteristics and treatment patterns among non-valvular atrial fibrillation patients: A real-world analysis in Dubai, United Arab Emirates. Open Med J 2019;6:33-41.

15. Dubai Drug code. Dubai Health Authority [cited February 14 and 15, 2020]; Available from: https://www.eclaimlink.ae/. [Last accessed on 2019 Dec 10].

16. Pisters R, Lane DA, Nieuwlaat R, de Vos CB, Crijns HJ, Lip GY. A novel user-friendly score (HAS-BLED) to assess 1-year risk of major bleeding in patients with atrial fibrillation: The Euro Heart Survey. Chest 2010;138:1093-100.

17. Deyo RA, Cherkin DC, Ciol MA. Adapting a clinical comorbidity index for use with ICD-9-CM administrative databases. J Clin Epidemiol 1992;45:613-9.

18. Brämer GR. International Statistical Classification of Diseases and related health problems. Tenth revision. World Health Stat Q 1988;41:32-6.

19. Thorwarth WT Jr. CPT: An open system that describes all that you do. J Am Coll Radiol 2008;5:555-60.

20. Cramer JA, Roy A, Burrell A, Fairchild CJ, Fuldeore MJ, Ollendorf DA, et al. Medication compliance and persistence: Terminology and definitions. Value Health 2008;11:44-7.
21. Andrade SE, Raebel MA, Morse AN, Davis RL, Chan KA, Finkelstein JA, et al. Use of prescription medications with a potential for fetal harm among pregnant women. Pharmacoepidemiol Drug Saf 2006;15:546-54.

22. Sikka R, Xia F, Aubert RE. Estimating medication persistency using administrative claims data. Am J Manag Care 2005;11:449-57.

23. Zubaid M, Rashed WA, Alsheikh-Ali AA, Almahmeed W, Shehab A, Sulaiman K, et al.; Gulf Survey of Atrial Fibrillation Events (Gulf SAFE) Investigators. Gulf Survey of Atrial Fibrillation Events (gulf SAFE): Design and baseline characteristics of patients with atrial fibrillation in the Arab Middle East. Circ Cardiovasc Qual Outcomes 2011;4:477-82.

24. Martinez C, Katholing A, Wallenhorst C, Granziera S, Cohen AT, Freedman SB. Increasing incidence of non-valvular atrial fibrillation in the UK from 2001 to 2013. Heart 2015;101:1748-54.

25. Proietti M, Marzona I, Vannini T, Tettamanti M, Fortino I, Merlino L, et al. Long-term relationship between atrial fibrillation, multimorbidity and oral anticoagulant drug use. Mayo Clin Proc 2019;94:2427-36.

26. Saheb Sharif-Askari N, Sulaiman SA, Saheb Sharif-Askari F, Al Sayed Hussain A, Tabatabai S, Al-Mulla AA. Hospitalized heart failure patients with preserved vs. reduced ejection fraction in Dubai, United Arab Emirates: A prospective study. Eur J Heart Fail 2014;16:454-60.

27. Gage BF, Waterman AD, Shannon W, Boechler M, Rich MW, Radford MJ. Validation of clinical classification schemes for predicting stroke: Results from the National Registry of Atrial Fibrillation. JAMA 2001;285:2864-70.

28. Kirchhof P, Radaideh G, Kim YH, Lanas F, Haas S, Amarenco P, et al.; Global XANTUS Program Investigators. Global prospective safety analysis of rivaroxaban. J Am Coll Cardiol 2018;72:141-53.

29. García Rodríguez LA, Martín-Pérez M, Vora P, Roberts L, Balabanova Y, Brobert $\mathrm{G}$, et al. Appropriateness of initial dose of non-vitamin $\mathrm{K}$ antagonist oral anticoagulants in patients with non-valvular atrial fibrillation in the UK. BMJ Open 2019;9:e031341.

30. Lackner TE, Battis GN. Use of warfarin for nonvalvular atrial fibrillation in nursing home patients. Arch Fam Med 1995;4:1017-26.

31. Rodríguez-Bernal CL, García-Sempere A, Hurtado I, Santa-Ana Y, Peiró S, Sanfélix-Gimeno G. Real-world adherence to oral anticoagulants in atrial fibrillation patients: A study protocol for a systematic review and meta-analysis. BMJ Open 2018;8:e25102.

32. Kim H, Lee YS, Kim TH, Cha MJ, Lee JM, Park J, et al. A prospective survey of the persistence of warfarin or NOAC in nonvalvular atrial fibrillation: A Comparison Study of Drugs for Symptom Control and Complication Prevention of Atrial Fibrillation (CODE-AF). Korean J Intern Med 2020;35:99-108.

33. Collings SL, Lefèvre C, Johnson ME, Evans D, Hack G, Stynes G, et al. Oral anticoagulant persistence in patients with non-valvular atrial fibrillation: A cohort study using primary care data in Germany. PLoS One 2017;12:e0185642.

34. Franchino-Elder J, Gilligan A, Song X, Hartaigh BO, Henriques C, SainskiNguyen A, et al. Healthcare resource utilization among non-valvular atrial fibrillation patients who switched from warfarin to a novel oral anti-coagulant. J Cardiol Cardiovasc Sci 2019;3:36-46.

35. Coleman CI, Peacock WF, Bunz TJ, Alberts MJ. Effectiveness and safety of apixaban, dabigatran, and rivaroxaban versus warfarin in patients with nonvalvular atrial fibrillation and previous stroke or transient ischemic attack. Stroke 2017;48:2142-9.

36. Hylek EM, Held C, Alexander JH, Lopes RD, De Caterina R, Wojdyla DM, et al. Major bleeding in patients with atrial fibrillation receiving apixaban or warfarin: The ARISTOTLE trial (Apixaban for Reduction in Stroke and Other Thromboembolic Events in Atrial Fibrillation): Predictors, characteristics, and clinical outcomes. J Am Coll Cardiol 2014;63:2141-7.

37. Gandhi SK, Salmon JW, Kong SX, Zhao SZ. Administrative databases and outcomes assessment: An overview of issues and potential utility. J Manag Care Spec Pharm 1999;5:215-22. 